\title{
Magnetic and electronic properties of spin-orbit coupled Dirac electrons on a (001) thin film of double-perovskite $\mathrm{Sr}_{2} \mathrm{FeMoO}_{6}$
}

\author{
Masahiko G. Yamada $\odot^{1,2,3,{ }^{*}}$ and George Jackeli ${ }^{3,4, \dagger}$ \\ ${ }^{1}$ Department of Materials Engineering Science, Osaka University, Toyonaka 560-8531, Japan \\ ${ }^{2}$ Institute for Solid State Physics, University of Tokyo, Kashiwa 277-8581, Japan \\ ${ }^{3}$ Max Planck Institute for Solid State Research, Heisenbergstrasse 1, D-70569 Stuttgart, Germany \\ ${ }^{4}$ Institute for Functional Matter and Quantum Technologies, University of Stuttgart, Pfaffenwaldring 57, D-70569 Stuttgart, Germany
}

(Received 20 December 2017; revised 3 May 2020; accepted 26 June 2020; published 21 July 2020)

\begin{abstract}
We present an interacting model for the electronic and magnetic behaviors of a strained (001) atomic layer of $\mathrm{Sr}_{2} \mathrm{FeMoO}_{6}$, which shows room-temperature ferrimagnetism and magnetoresistance with potential spintronics application in the bulk. We find that the strong spin-orbit coupling in the molybdenum $4 d$ shell gives rise to a robust ferrimagnetic state with an emergent spin-polarized electronic structure consisting of flatbands and four massive or massless Dirac dispersions. Based on the spin-wave theory, we demonstrate that the magnetic order remains intact for a wide range of doping, leading to the possibility of exploring flatband physics, such as Wigner crystallization in electron-doped $\mathrm{Sr}_{2-x} \mathrm{La}_{x} \mathrm{FeMoO}_{6}$.
\end{abstract}

DOI: 10.1103/PhysRevMaterials.4.074007

\section{INTRODUCTION}

The coexistence of a strong spin-orbit coupling (SOC) and low dimensionality gives rise to interesting phases of matter [1]. Two-dimensional (2D) systems confined in atomically thin films can possess rich electronic properties different from the bulk and could host various correlated phenomena. Especially, the rapid progress in synthesizing atomic-scale slabs, superlattices, and heterostructures of correlated transitionmetal oxides by pulsed laser deposition or molecular beam epitaxy has motivated the exploration of various (perovskite) compounds epitaxially grown on different cubic substrates as potential nanoscale devices [1-4]. An advantage of the epitaxial growth is that by changing the substrate, one can introduce strain to thin films due to a mismatch of the lattice constants and thereby control the electronic state, which we call strain engineering [5]. By replacing $3 d$ transition-metal ions with heavier $4 d$ or $5 d$ ions, one can even control the strength of the SOC. These flexibilities of epitaxially grown atomic-scale layers could pave a way to search for unusual spin-orbit-coupled correlated phenomena in 2D systems. In this context, theoretical exploration of possible phases can provide useful guidance.

To explore such collective phenomena, perovskite oxide is one of the best-established platforms [1]. For instance,

\footnotetext{
*myamada@mp.es.osaka-u.ac.jp

${ }^{\dagger}$ Also at Andronikashvili Institute of Physics, 0177 Tbilisi, Georgia.
}

Published by the American Physical Society under the terms of the Creative Commons Attribution 4.0 International license. Further distribution of this work must maintain attribution to the author(s) and the published article's title, journal citation, and DOI. Open access publication funded by the Max Planck Society. the $\mathrm{LaAlO}_{3} / \mathrm{SrTiO}_{3}(\mathrm{LAO} / \mathrm{STO})$ interface is known as a good 2D electron gas system [6] and hosts various electronic phenomena, including 2D superconductivity [7] and Rashba SOC effects [8]. Although there have appeared different types of atomic-scale 2D systems like transition-metal dichalcogenides [9], where we can expect a stronger effect of SOC than in graphene [10], perovskite systems are still important playgrounds because the knowledge on their synthesis and chemical properties has been accumulated for a long time.

Specifically, the bulk double perovskite compounds, such as $\mathrm{Sr}_{2} \mathrm{FeMoO}_{6}$ (SFMO) and $A_{2} \mathrm{FeMoO}_{6}(A=\mathrm{Ca}, \mathrm{Ba}, \mathrm{Pb})$, have been investigated intensely as examples of half-metallic ferrimagnets (FiMs) with enhanced magnetoresistance at room temperature and as possible spintronics devices based on the high spin polarization of charge carriers [11-14]. Theoretical studies of the carrier-induced FiM in cubic SFMO have been previously discussed within the ab initio [15-18] and model Hamiltonian approaches [19-21] without including the SOC.

Since double perovskite compounds have a two-sublattice structure, the synthesis of high-quality SFMO thin films with completely staggered $\mathrm{Fe} / \mathrm{Mo}$ sublattices is experimentally challenging. Here, motivated by a recent fabrication of wellordered thin films of double perovskite SFMO epitaxially grown along the (001) direction on various perovskite substrates that showed a ferrimagnetic ground state [22], we theoretically explore the combined effects of the strong SOC, tetragonal elongation, and carrier doping in a (001) layer of SFMO. For example, a typical perovskite substrate STO has a slightly shorter lattice constant $(\sim-1.1 \%)$ than SFMO, so STO/SFMO heterostructures [23] would be ideal systems to investigate such effects.

In insulating compounds such as $\mathrm{Ba}_{2} \mathrm{YMoO}_{6}$, the SOC locally stabilizes the $j=3 / 2$ quartet of $\mathrm{Mo}^{5+}$ and triggers rich multiorbital physics [24-26], unlike in the insulating iridates, where the orbital shape of the lowest energy $j=1 / 2$ 
state is fixed, and the SOC manifests itself in the anisotropic exchange interactions $[27,28]$. On the other hand, in SFMO, the molybdenum $4 d$ electrons are itinerant, forming a conduction band. In this case, a strong impact of the SOC on the band structure is expected. Indeed, it has recently been proposed that the SOC may stabilize a Chern insulator phase in the (001) and (111) monolayers of double perovskites $[29,30]$ and lead to a topologically nontrivial band structure in $\mathrm{BaTiO}_{3} / \mathrm{Ba}_{2} \mathrm{FeReO}_{6} / \mathrm{BaTiO}_{3} 2 \mathrm{D}$ quantum wells [31]. It should be noted that the tight-binding model for this system with the SOC and tetragonal compression has been investigated [30] in the free fermion level. However, the effect of carrier doping on the magnetism in the presence of the SOC is still illusive.

In this paper, we study a (001) layer of pure and doped SFMO based on a minimal microscopic model within a large$S$ expansion. We find that the strong SOC gives rise to a robust nontrivial magnetic state with an electronic structure consisting of four spin-polarized massive or massless Dirac dispersions as well as flatbands. Based on the spin-wave theory, we demonstrate that such an unusual magnetic state is stable in a large, experimentally relevant range of carrier doping. In the electron-doped $\mathrm{Sr}_{2-x} \mathrm{La}_{x} \mathrm{FeMoO}_{6}$, we suggest that the extra electrons would occupy a fully polarized flatband.

\section{MODEL}

In the (001) layer of double perovskite SFMO, Fe and Mo ions form a checkerboard pattern on a square lattice [see Fig. 1(a)], and these metal ions reside inside the oxygen octahedra. In the ionic picture, iron is in the $\mathrm{Fe}^{3+}$ valence state with five $3 d$ electrons coupled by Hund's rule into the highspin state forming a localized $S=5 / 2$ moment. The $\mathrm{Mo}^{5+}$ ion has a single $4 d$ electron in the $t_{2 g}$ manifold of degenerate $x y$, $x z$, and $y z$ orbitals. The lowest energy coherent charge transfer process takes place when this single electron moves from $\mathrm{Mo}^{5+}$ to a neighboring $\mathrm{Fe}^{3+}$ through the hybridization between the same $t_{2 g}$ states along a given bond. For example, on a bond along the $x$ direction, there is a finite overlap either between $x y$ or $x z$ neighboring orbitals with a real amplitude $-t$.

We consider a tetragonal elongation of the oxygen octahedra due to a substrate-induced compressive strain. The corresponding tetragonal crystal field $\Delta_{\mathrm{T}}>0$ lifts the threefold $t_{2 g}$ orbital degeneracy by stabilizing an orbital doublet of the axial $x z$ and $y z$ orbitals and by placing the planar $x y$ orbital at a higher energy. It should be noted that for the $x y$ orbital, where the SOC is completely quenched by the tetragonal distortion, we can repeat the analysis of the cubic case without the SOC [20] to discuss the stability against doping. In addition, we include the $\mathrm{SOC} \lambda>0$ in $\mathrm{Mo}^{5+}$ that further lifts the degeneracy of the $x z$ and $y z$ orbitals by stabilizing $j^{z}= \pm 3 / 2$ Kramers doublet of the effective total angular momentum $j=s+l=3 / 2$ quartet [32]. Here, $s=$ $1 / 2$ and $l=1$ are spin and effective angular momentum of a $t_{2 g}$ electron, respectively [33]. We will not include the SOC for the $\mathrm{Fe} 3 d$ orbitals because it is much weaker than that for the Mo $4 d$ orbitals, and, moreover, it is fully quenched in the high-spin state of $\mathrm{Fe}^{3+}$. The resulting local energy structure of $\mathrm{Mo}^{5+}$ is depicted in Fig. 1(c), and the explicit forms of (a)

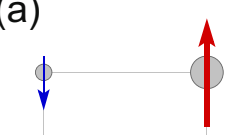

(b)

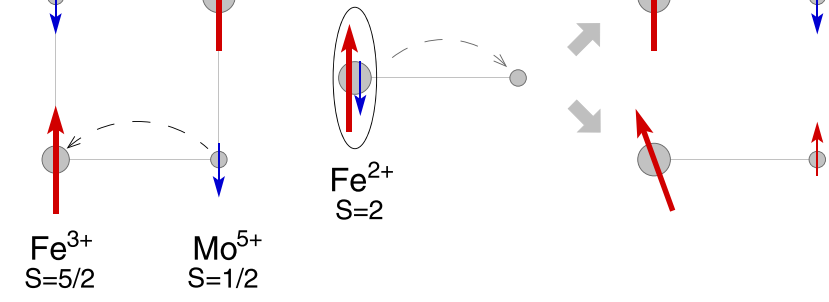

(c)

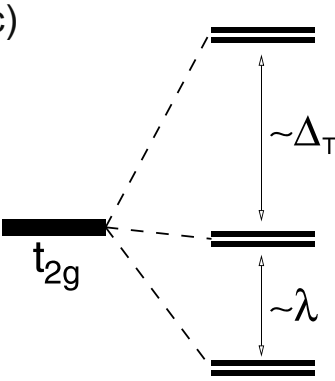

(d)

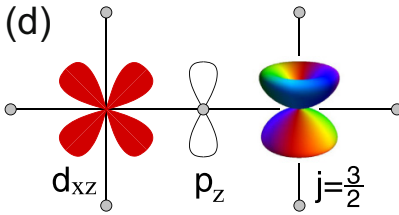

(e)

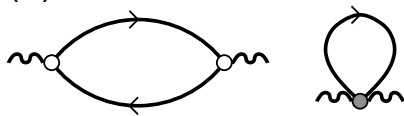

FIG. 1. (a) Ferrimagnetic order of the $\mathrm{Fe}^{3+}\left(3 d^{5}\right) S=5 / 2$ local moments (long arrows) and the $\mathrm{Mo}^{5+}\left(4 d^{1}\right) s=1 / 2$ states (short arrows) in the ionic picture. Electron transfer from $\mathrm{Mo}^{5+}$ to $\mathrm{Fe}^{3+}$ is only allowed when its spin is antiparallel to the localized moment, otherwise blocked by the Pauli exclusion principle. (b) When an electron moves from the $\mathrm{Fe}^{2+} S=2$ state, an entangled state of the local and carrier spins may leave behind the local moment polarized along the ordered direction (top) or polarized away from it by creating a single magnon state behind (bottom). (c) Splitting of the $t_{2 g}$ level by a tetragonal crystal field $\Delta_{\mathrm{T}}$ and the SOC $\lambda$ into three Kramers pairs. (d) Hopping pathway between the Fe $3 d_{x z}$ orbital (left) and the Mo $j^{z}=3 / 2$ state (right) via the oxygen $2 p_{z}$ orbital. (e) Magnon self-energies in the leading order of the $1 / S$ expansion. Solid (wavy) lines represent fermion (magnon) propagators. White (grey) vertices correspond to the coupling between the magnon and the transverse (longitudinal) particle-hole excitations.

$j^{z}= \pm 3 / 2$ wave functions are given by

$$
\begin{array}{r}
\left|j^{z}=\frac{3}{2}\right\rangle=-\frac{1}{\sqrt{2}}\left(i\left|d_{x z \uparrow}\right\rangle+\left|d_{y z \uparrow}\right\rangle\right) \equiv\left|c_{\uparrow}\right\rangle, \\
\left|j^{z}=-\frac{3}{2}\right\rangle=-\frac{1}{\sqrt{2}}\left(i\left|d_{x z \downarrow}\right\rangle-\left|d_{y z \downarrow}\right\rangle\right) \equiv\left|c_{\downarrow}\right\rangle,
\end{array}
$$

and are labeled hereafter by fermionic annihilation operators $c_{\sigma}$ with a pseudospin $\sigma=\uparrow, \downarrow$.

Here we take the limit of a strong SOC $\lambda$ and a tetragonal field $\Delta_{\mathrm{T}}$ compared to the nearest-neighbor (NN) hopping amplitude $|t|$. Projecting the $t_{2 g}$ orbitals onto the lowest energy states Eqs. (1), we have obtained a low-energy Hamiltonian for a charge transfer between $\mathrm{NN} \mathrm{Fe}$ and Mo ions [see Fig. 1(d)], as follows:

$$
\begin{aligned}
\mathcal{H}_{t}= & \frac{t}{\sqrt{2}}\left[\sum_{\langle i j\rangle \in x, \sigma} i d_{i, x z \sigma}^{\dagger} c_{j, \sigma}+\sum_{\langle i j\rangle \in y, \sigma} \sigma d_{i, y z \sigma}^{\dagger} c_{j, \sigma}+\text { H.c. }\right] \\
& +\Delta\left[\sum_{i} n_{i}^{(d)}-\sum_{j} n_{j}^{(c)}\right],
\end{aligned}
$$


where $i(j)$ labels $\mathrm{Fe}(\mathrm{Mo})$ ions, $\langle i j\rangle \in x(y)$ refers to each NN bond along the $x(y)$ direction, $\sigma=\uparrow, \downarrow= \pm 1$ stands for a spin index, $2 \Delta$ is a charge transfer gap between $\mathrm{Mo}^{5+}$ and $\mathrm{Fe}^{3+}$, the number operators $n_{i}^{(d)}$ and $n_{j}^{(c)}$ measure carrier density $d_{i}^{\dagger} d_{i}$ and $c_{j}^{\dagger} c_{j}$, respectively. In the undoped SFMO, there is one carrier $n=n^{(d)}+n^{(c)}=1$ per formula unit, ignoring the localized half-filled $\mathrm{Fe} d$ shell. The SOC manifests itself in the spin-dependent hopping in Eq. (2) that explicitly breaks the original $S U(2)$ symmetry. Hereafter, we set $t=1$ as the energy scale for simplicity.

When an itinerant electron visits the $\mathrm{Fe}^{3+}$ ion with a core spin $S=5 / 2$, the resulting total spin $\mathcal{S}$ of $\mathrm{Fe}^{2+}$ could, in principle, take one of the two possible values $\mathcal{S}=2$ and $\mathcal{S}=3$. However, the maximum allowed spin quantum number for six electrons in a $d$ shell is $\mathcal{S}=2$. The unphysical $\mathcal{S}=3$ states appear because the local and itinerant spin operators on the Fe site are treated as independent variables. To project the enlarged Hilbert space onto the physical one, we supplement the hopping Hamiltonian Eq. (2) by a local antiferromagnetic coupling $J \rightarrow \infty$ between the local and itinerant spins [20]. The total Hamiltonian then becomes $\mathcal{H}=\mathcal{H}_{t}+\mathcal{H}_{\mathcal{S}}$, where

$$
\mathcal{H}_{\mathcal{S}}=J \sum_{i}\left[\vec{S}_{i} \cdot \vec{s}_{i}+\frac{S+1}{2} n_{i}^{(d)}\right] .
$$

The sum is taken over every Fe site $i$, and $\vec{S}_{i}$ and $\vec{s}_{i}$ are operators for the local and itinerant spins, respectively.

\section{FERRIMAGNETIC GROUND STATE}

The model defined by Eqs. (2) and (3) is one version of canonical double exchange (DE) problems with an infinite exchange coupling between the local and itinerant spins. Similarly to the DE, a maximum kinetic energy gain is achieved when the local moments align ferromagnetically (FM) and, in the present case, antiparallel to the itinerant spins, giving rise to an FiM state.

We consider the FiM order along the tetragonal symmetry $z$ axis and later discuss its stability within the large- $S$ spin-wave theory. We introduce fermionic operators $D_{\downarrow(\uparrow)}$ for the carriers on the Fe sites with their spins quantized along the local moments. This representation diagonalizes the spin part of the Hamiltonian Eq. (3) and projects out the fermionic states $D_{\uparrow}$ corresponding to the unphysical states with $\mathcal{S}=3$ (see Ref. [20] for details). The $d$ operators in Eq. (2) in terms of the new ones are expressed as $d_{x z(y z) \downarrow}=D_{x z(y z) \downarrow}\left[1-b^{\dagger} b /(4 S)\right]$ and $d_{x z(y z) \uparrow}=-D_{x z(y z) \downarrow} b^{\dagger} / \sqrt{2 S}$, where $b$ is a bosonic annihilation operator for a single magnon state. This is created when a spin-down electron moves away from $\mathrm{Fe}^{2+}$, which is in the entangled $\mathcal{S}=2$ state of the local and carrier spins, leaving an $\mathrm{Fe}^{3+}$ local moment in the $S^{z}=S-1=3 / 2$ single magnon state which is tilted away from the fully polarized $S=$ $S^{z}=5 / 2$ state [see Fig. 1(b)]. This representation provides the correct matrix elements of fermionic operators within the eigenstates of the allowed total spin, $S=5 / 2$ and $\mathcal{S}=2$, in the perturbative level and retains a quantum nature of the local moments. The large- $S$ expansion is justified by the smallness of $1 / S=2 / 5$. Indeed, the next-order contribution of $1 / S^{2}$ is irrelevant for a small doping level in the previous calculation [20], and thus we expect that the expansion until $1 / S$ is enough

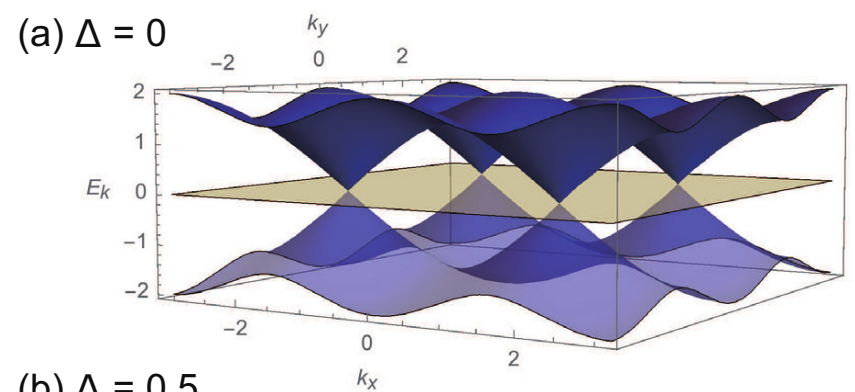

(b) $\Delta=0.5$

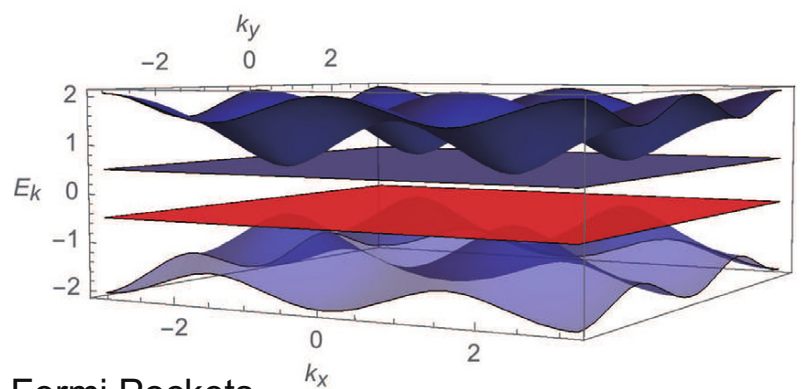

(c) Fermi Pockets

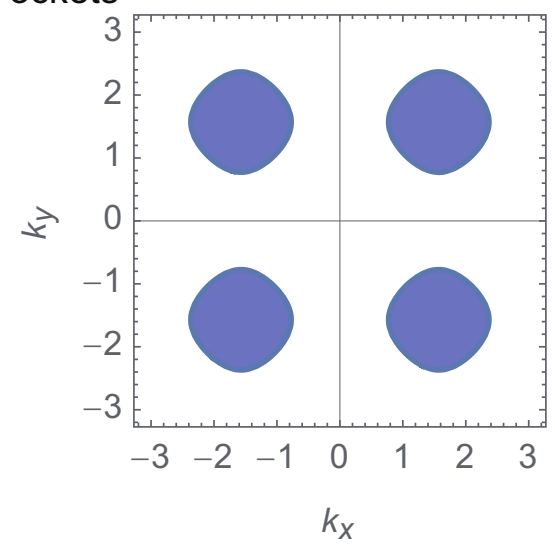

FIG. 2. Band structure of the single-electron Hamiltonian Eq. (4) for (a) gapless $\Delta=0$ and (b) gaped $\Delta \neq 0$ cases (see the text for the nature of the bands). (c) Fermi pockets of the lowest band for a carrier density $n=0.75$ per formula unit with $\Delta=0$.

in our case. The approximation to include a strong SOC in Mo and no SOC in Fe can be justified easily by the fact that SOC is completely quenched in spherical half-filled $d$ orbitals of $\mathrm{Fe}^{3+}$, while SOC is active in the $t_{2 g}$ orbitals of Mo [34].

Inserting the above representation into Eq. (2), we get $\mathcal{H}=\mathcal{H}_{0}+\mathcal{H}_{1}+\mathcal{H}_{2}$, where $\mathcal{H}_{0}$ is a single-particle part and $\mathcal{H}_{1(2)} \sim 1 / \sqrt{S}(1 / S)$ refers to the coupling of the magnons with the transverse (longitudinal) particle-hole pairs.

Diagonalizing the noninteracting $\mathcal{H}_{0}$ part, we get the following expression in the momentum space:

$$
\mathcal{H}_{0}=\sum_{k}\left\{\Delta\left[\alpha_{k \downarrow}^{\dagger} \alpha_{k \downarrow}-c_{k \uparrow}^{\dagger} c_{k \uparrow}\right]+E_{k}\left[\beta_{k \downarrow}^{\dagger} \beta_{k \downarrow}-\gamma_{k \downarrow}^{\dagger} \gamma_{k \downarrow}\right]\right\},
$$

where $E_{k}=\sqrt{\Delta^{2}+2\left(\cos ^{2} k_{x}+\cos ^{2} k_{y}\right)}$ and the eigenstates $\alpha_{k \downarrow}, \beta_{k \downarrow}, \gamma_{k \downarrow}$, and $c_{k \uparrow}$ have been obtained by a unitary transformation. The band structure Eq. (4) is composed of four bands and is shown in Figs. 2(a)-2(b). The two flat $\alpha_{\downarrow}$ and $c_{\uparrow}$ bands correspond to a nonbonding state composed of the 
(a)

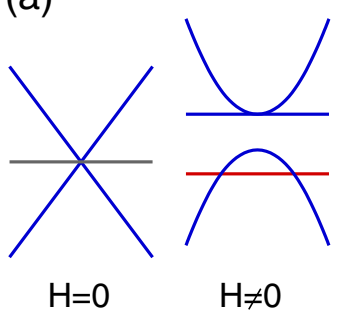

(b)

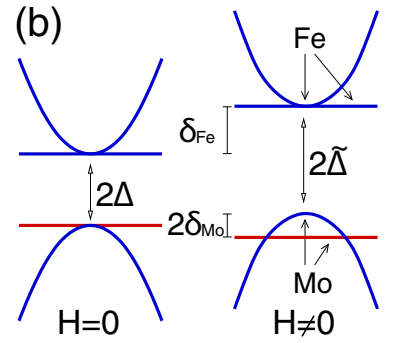

FIG. 3. Schematic view of the band structure renormalization induced by an applied magnetic field $(H)$ for (a) gapless $\Delta=0$ and (b) gapped $\Delta \neq 0$ cases. Spin-up, spin-down, and spin-unpolarized states are marked by red, blue, and grey colors, respectively. The spin-down (spin-up) flatband has a purely Fe (Mo) character. The higher-(lower-) energy dispersive band at its minima (maxima) is solely made of the Fe (Mo) states. In both cases, (a) and (b), an applied field shifts up the higher-energy Fe bands by $\delta_{\mathrm{Fe}}=g_{\mathrm{Fe}} \mu_{B} H / 2$ and splits the lower-energy Mo flat and dispersive bands by $2 \delta_{\mathrm{Mo}}=$ $g_{\mathrm{Mo}} \mu_{B} H$. Here, $g_{\mathrm{Fe}} \simeq 2$ and $g_{\mathrm{Mo}} \ll 1$ are the corresponding $g$ factors [35], and $2 \tilde{\Delta}=2 \Delta+\left(g_{\mathrm{Fe}}-g_{\mathrm{Mo}}\right) \mu_{B} H / 2$ is a renormalized mass gap.

$d_{x z \downarrow}$ and $d_{y z \downarrow}$ orbitals of Fe and a localized $j^{z}=3 / 2$ state of Mo, respectively. The dispersive antibonding $\beta_{\downarrow}$ and bonding $\gamma_{\downarrow}$ bands are made of spin-down states of Mo and Fe. The next-nearest-neighbor (NNN) hopping between the same Fe (or Mo) ions, not considered here, might in principle give a finite dispersion to the flatbands. However, the corresponding hopping is between the $d_{x z}$ and $d_{y z}$ orbitals, and is extremely small ( $\sim$ few meV) [29]. Moreover, it exactly vanishes when projected onto the complex wave functions of the Mo $j^{z}=$ $\pm 3 / 2$ states due to a destructive quantum interference. Notice that the robust half metallicity and the flatband owe the halffilled $d$ orbitals of $\mathrm{Fe}^{3+}$, which is different from $\mathrm{Cr}^{3+}$ in Ref. [30].

We now discuss the effects of a uniform external magnetic field $H$ on $\mathcal{H}_{0}$. This just splits the four bands without hybridization because $\mathcal{H}_{0}$ conserves the $z$ component of the real spins. While the external field is uniform, the Zeeman splitting $g \mu_{B} H$ of the itinerant electrons on the $\mathrm{Fe}$ and $\mathrm{Mo}$ ions become staggered due to the difference in the $g$ factors. As shown in Fig. 3, this would allow us to control the mass of Dirac dispersions and also to dope the flatband just above the Fermi energy.

The flatbands, which are already fully spin polarized, are different from the unpolarized ones, such as the ones in the (110) thin films of STO [36] or in (metal)-organic systems [37,38], supporting the flatband ferromagnetism [39-41]. Therefore, in electron-doped $\mathrm{Sr}_{2-x} \mathrm{La}_{x} \mathrm{FeMoO}_{6}$ or SFMO under a strong magnetic field, where extra electrons occupy the nondispersive Mo band, we anticipate other types of instabilities, such as Wigner crystallization [42] or various types of complex charge-ordered patterns, as well as the formation of self-trapped polaronic states of minority spins at the Mo sites. As confirmed in the following section, the FiM state is stable in a wide carrier doping range of the electron doping, and, consequently, the minority-spin flat Mo band can indeed be electron doped.

(a)

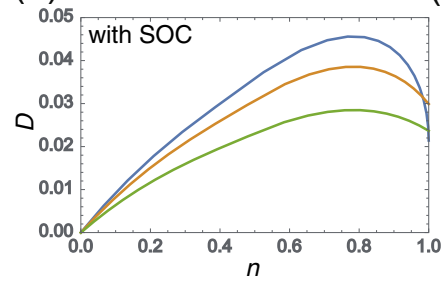

(b)

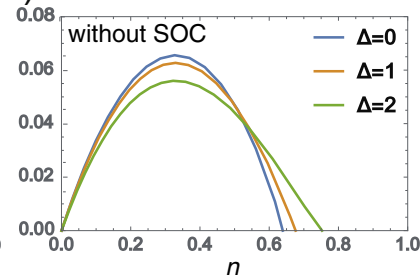

FIG. 4. (a) Spin stiffness $\mathcal{D}$ of the FiM ordered state versus carrier density $n$ for $0<n<1$ at various values of the gap $\Delta$. For $1<n<2$ (not shown), the spin stiffness remains nearly constant. (b) For comparison, same as (a) but without SOC [20].

\section{SPIN-WAVE SPECTRUM}

We now analyze the stability of the FiM order state postulated above. To this end, we derive a spin-wave excitation spectrum from the magnon Green's function $G_{q, \omega}=1 /[\omega-$ $\Sigma_{q, \omega}$ ] evaluated within the leading order of the large- $S$ expansion. First, we note that in the classical $S \rightarrow \infty$ limit, the magnons are localized, and they become dispersive only due to quantum corrections. The corresponding magnon selfenergy corrections $\left(\Sigma_{q, \omega} \sim 1 / S\right)$, shown in Fig. 1(c), stem from magnon interactions with propagating transverse and longitudinal particle-hole excitations. Their expressions are quite lengthy and given in the Appendix. We find that a coherent spin-wave mode emerges below the Stoner continuum with the following dispersion relation in the low-energy limit:

$$
\omega_{q}=4 J_{1} S\left[1+\Gamma_{1 q}\right]+8 J_{2} S\left[1-\Gamma_{2 q}\right],
$$

where $\Gamma_{1 q}=\cos q_{x} \cos q_{y}, \Gamma_{2 q}=\left(\cos ^{2} q_{x}+\cos ^{2} q_{y}\right) / 2, J_{1}$, and $J_{2}$ are the carrier-induced exchange couplings between the NN and NNN local moments, respectively. They depend only on the carrier density and the charge transfer gap $\Delta$. We note that the ferromagnetic state of the localized moments is the exact ground state of the effective anisotropic spin-only Hamiltonian [see Appendix D].

The spectrum Eq. (5) is gapless at $q=(\pi, 0)$ and at the symmetry-related points, which is very surprising because (i) the model defined by Eqs. (2) and (3) does not host any apparent continuous spin-rotation symmetry, and (ii) the gapless points are away from the FM Bragg point $q=(0,0)$. Actually, the model has a hidden $S U(2)$ symmetry that can be uncovered by a gauge transformation.

For the spectrum Eq. (5), the spin stiffness of the FiM ordered state is given by $\mathcal{D}=2 J_{1} S+4 J_{2} S$. Shown in Fig. 4(a) is the dependence of $\mathcal{D}$ on the carrier density $n$ for $0<n<1$ at various values of the band gap $\Delta$. In the range $1<n<2$, $\mathcal{D}$ remains constant for $\Delta=0$. This is because the added electron carriers occupy the unpolarized flatband, and no additional potential or kinetic energy is gained. For $\Delta \neq 0$, $\mathcal{D}$ becomes very weakly renormalized ( $\sim$ few percent) as carriers occupy the minority spin flatband. For comparison, in Fig. 4(b) we plot the spin stiffness obtained at a zero SOC $[20]$. $\mathcal{D}$ vanishes at some critical doping, signaling the instability of the FiM order. Without SOC, the FiM ground state cannot be stabilized at $n=1$ or $n$ slightly larger than 1 .

Thus, a strong SOC extends the stability window of the FiM order to the experimentally accessible electron-doping 
range. The reason behind the extended stability is the SOCinduced electronic band-structure reconstruction, which transforms a large Fermi surface centered around the $q=(0,0)$ to four small Fermi pockets around $\left( \pm \frac{\pi}{2}, \pm \frac{\pi}{2}\right)$, as shown in Fig. 2(c), allowing more kinetic energy gain with an increasing carrier density. Indeed, as seen experimentally [43], electron-doped $\mathrm{Sr}_{2-x} \mathrm{La}_{x} \mathrm{FeMoO}_{6}$ thin films do exhibit stable FiM order in a wide range of doping as correctly shown here by the model with the SOC. We note that the disorder effect in $\mathrm{Sr}_{2-x} \mathrm{La}_{x} \mathrm{FeMoO}_{6}$ does not affect the flatband as electrons in the flatband are already localized.

In conclusion, we have proven the stability of the FiM ground state of SFMO thin films against doping within a perturbative analysis. We discovered that the SOC plays a critical role in the enhancement of the stability and results in a phase with an unusual band structure, including Dirac dispersions and flatbands. We anticipate that this gives rise to interesting collective behaviors, such as Wigner crystallization [42]. The disorder effect in a Wigner crystal has also been discussed in Ref. [44] as a pinned Wigner crystal.

The above-proposed nontrivial band structure in this quasi2D system can be directly verified through angle-resolved photoemission spectroscopy (ARPES), and we believe that our theoretical findings will motivate future ARPES experiments.

\section{ACKNOWLEDGMENTS}

We wish to acknowledge A. F. Bangura, J. A. N. Bruin, A. M. Cook, D. Hirai, I. H. Inoue, J. Lee, J. Romhányi, A. Paramekanti, D. D. Sarma, S. Tsuneyuki, T. Vekua, M. Oshikawa, and especially H. Takagi for illuminating discussions. We thank A. Subedi for the critical reading of the paper and comments. M.G.Y. acknowledges the opportunity of his internship and a kind hospitality at the Quantum Materials Department, Max Planck Institute for Solid State Research, Stuttgart, Germany, where the major part of this work was done. A part of this work was completed at MPI-PKS international workshop "Topological Phenomena in Novel Quantum Matter" and at KITP, UCSB, supported by the US National Science Foundation under Grants No. NSF PHY11-25915 and No. PHY-1748958. This work was also supported by JST CREST Grant No. JPMJCR19T5, Japan, by JSPS KAKENHI Grants No. JP15H02113 and No. JP17J05736, and by JSPS Strategic International Networks Program No. R2604 TopoNet. M.G.Y. is supported by the Materials Education program for the future leaders in Research, Industry, and Technology (MERIT) and by JSPS. We also acknowledge the support of the Max-Planck-UBC-UTokyo Centre for Quantum Materials.

\section{APPENDIX A: DIAGONALIZATION OF THE FREE-FERMION PART}

The eigenstates of the tight-binding model are obtained by a unitary transformation as

$$
\begin{aligned}
& \alpha_{k \downarrow}=-i v_{k} D_{x z, k \downarrow}+u_{k} D_{y z, k \downarrow}, \\
& \beta_{k \downarrow}=\tilde{u}_{k} \tilde{D}_{k \downarrow}-\tilde{v}_{k} e^{i k_{x}} c_{k \downarrow},
\end{aligned}
$$

$$
\begin{aligned}
\gamma_{k \downarrow} & =\tilde{v}_{k} \tilde{D}_{k \downarrow}+\tilde{u}_{k} e^{i k_{x}} c_{k \downarrow}, \\
\tilde{D}_{k \downarrow} & =i u_{k} D_{x z, k \downarrow}+v_{k} D_{y z, k \downarrow},
\end{aligned}
$$

with the following factors: $u_{k}=T_{x, k} / \varepsilon_{k}, \quad v_{k}=T_{y, k} / \varepsilon_{k}$, $\tilde{u}_{k}=\sqrt{\left(1+\Delta / E_{k}\right) / 2}, \quad \tilde{v}_{k}=\sqrt{\left(1-\Delta / E_{k}\right) / 2}$, and $T_{x(y), k}=$ $\sqrt{2} \cos k_{x(y)}$, where $E_{k}=\sqrt{\varepsilon_{k}^{2}+\Delta^{2}}$ and $\varepsilon_{k}=\sqrt{T_{x, k}^{2}+T_{y, k}^{2}}$.

\section{APPENDIX B: MAGNON-FERMION INTERACTION}

The interaction between the magnons and the transverse and longitudinal components of particle-hole excitations is described by the following $\mathcal{H}_{1} \sim 1 / \sqrt{S}$ and $\mathcal{H}_{2} \sim 1 / S$ terms, respectively:

$$
\begin{aligned}
\mathcal{H}_{1}= & \frac{1}{\sqrt{2 S N}} \sum_{k, q}\left\{c _ { k - q \uparrow } ^ { \dagger } \left[L_{k, q} \alpha_{k \downarrow}+M_{k, q} \beta_{k \downarrow}\right.\right. \\
& \left.\left.+N_{k, q} \gamma_{k \downarrow}\right] b_{q}^{\dagger} e^{i\left(q_{x}-k_{x}\right)}+\text { H.c. }\right\}, \\
\mathcal{H}_{2}= & \frac{1}{4 S N} \sum_{k, p, q} b_{q}^{\dagger} b_{q+k-p}\left\{P_{k, p}^{\alpha \beta} \alpha_{k \downarrow}^{\dagger} \beta_{p \downarrow}+P_{p, k}^{\alpha \beta} \beta_{k \downarrow}^{\dagger} \alpha_{p \downarrow}\right. \\
+ & P_{k, p}^{\alpha \gamma} \alpha_{k \downarrow}^{\dagger} \gamma_{p \downarrow}+P_{p, k}^{\alpha \gamma} \gamma_{k \downarrow}^{\dagger} \alpha_{p \downarrow}+P_{k, p}^{\beta \beta} \beta_{k \downarrow}^{\dagger} \beta_{p \downarrow} \\
+ & \left.P_{k, p}^{\beta \gamma} \beta_{k \downarrow}^{\dagger} \gamma_{p \downarrow}+P_{p, k}^{\beta \gamma} \gamma_{k \downarrow}^{\dagger} \beta_{p \downarrow}+P_{k, p}^{\gamma \gamma} \gamma_{k \downarrow}^{\dagger} \gamma_{p \downarrow}\right\},
\end{aligned}
$$

with interaction vertices

$$
\begin{aligned}
L_{k, q} & =-T_{x, k-q} v_{k}-T_{y, k-q} u_{k}, \\
M_{k, q} & =T_{x, k-q} u_{k} \tilde{u}_{k}-T_{y, k-q} v_{k} \tilde{u}_{k}, \\
N_{k, q} & =T_{x, k-q} u_{k} \tilde{v}_{k}-T_{y, k-q} v_{k} \tilde{v}_{k}, \\
P_{k, p}^{\alpha \beta} & =T_{x, p} v_{k} \tilde{v}_{p}-T_{y, p} u_{k} \tilde{v}_{p}, \\
P_{k, p}^{\alpha \gamma} & =-T_{x, p} v_{k} \tilde{u}_{p}+T_{y, p} u_{k} \tilde{u}_{p}, \\
P_{k, p}^{\beta \beta} & =-\left[\left(T_{x, p} u_{k}+T_{y, p} v_{k}\right) \tilde{u}_{k} \tilde{v}_{p}+(k \leftrightarrow p)\right], \\
P_{k, p}^{\beta \gamma} & =\left(T_{x, p} u_{k}+T_{y, p} v_{k}\right) \tilde{u}_{k} \tilde{u}_{p}-(k \leftrightarrow p, \tilde{u} \leftrightarrow \tilde{v}), \\
P_{k, p}^{\gamma \gamma} & =\left(T_{x, p} u_{k}+T_{y, p} v_{k}\right) \tilde{v}_{k} \tilde{u}_{p}+(k \leftrightarrow p),
\end{aligned}
$$

where $(k \leftrightarrow p)$ means the corresponding term with $k$ exchanged for $p$.

\section{APPENDIX C: DETAILS OF THE SPIN-WAVE SPECTRUM}

The spin-wave spectrum is derived from the poles of the magnon Green's function $G_{q, \omega}=1 /\left[\omega-\Sigma_{q, \omega}\right]$, where the magnon self-energy $\Sigma_{q, \omega}$ is composed of two parts within the leading order of $1 / S$ expansion. They are diagrammatically depicted in Fig. 1(c) in the main text and can be grouped into the following algebraic form when $0<n<2$ :

$$
\begin{aligned}
\Sigma_{q, \omega}= & \frac{1}{2 S N} \sum_{k}\left\{\left|L_{k, q}\right|^{2} \frac{n_{c, k-q \uparrow}-n_{\alpha, k \downarrow}}{\omega-2 \Delta}\right. \\
& \left.+\left|M_{k, q}\right|^{2} \frac{n_{c, k-q \uparrow}-n_{\beta, k \downarrow}}{\omega-E_{k}-\Delta}+\left|N_{k, q}\right|^{2} \frac{n_{c, k-q \uparrow}-n_{\gamma, k \downarrow}}{\omega+E_{k}-\Delta}\right\} \\
& +\frac{1}{4 S N} \sum_{k} P_{k, k}^{\gamma \gamma} n_{\gamma, k \downarrow} .
\end{aligned}
$$


Here, $n_{m, k \sigma}$ is the occupation number of the $m_{k \sigma}$ state $(m=$ $\alpha, \beta, \gamma, c)$ depending on the total carrier density and $N$ is the number of unit cells.

From the poles of $G$, we got a Stoner continuum and a gapless mode of the spin wave in the order of $1 / S$. Assuming $|\omega|$ to be small, the obtained low-energy spin wave has a form of a localized spin model with anisotropic $\mathrm{NN}$ and isotropic NNN exchange couplings, $J_{1}$ and $J_{2}$, respectively (see the next section). For the carrier density $n \leqslant 1$, only the lowest dispersive mode is occupied and exchange couplings, $J_{1}$ and $J_{2}$, are given by

$$
\begin{aligned}
& J_{1}=\frac{1}{8 S^{2} N} \sum_{k} T_{x, k}^{2} T_{y, k}^{2} \frac{n_{\gamma, k \downarrow}}{E_{k} \varepsilon_{k}^{2}}, \\
& J_{2}=\frac{1}{8 S^{2} N} \sum_{k} \cos \left(2 k_{x}\right) T_{x, k}^{2} \frac{n_{\gamma, k \downarrow}}{E_{k} \varepsilon_{k}^{2}} .
\end{aligned}
$$

For the filling $n>1$, we need to treat the case $\Delta=0$ and the case $\Delta>0$ separately. When $\Delta=0$, the band structure consists of Dirac-like dispersions and two degenerate flatbands $\alpha$ and $c$, with opposite spin polarization [see Fig. 2(a) in the main text]. Due to the degeneracy $n_{c, k-q \uparrow}=n_{\alpha, k \downarrow}$, the first Lindhard function in Eq. (C1) vanishes, and we can then take the approximation $\omega / E_{k} \rightarrow 0$. We can easily find that the sum of the second and third Lindhard functions also vanish other than the term including $n_{\gamma, k \downarrow}$ in this low-energy limit, so the spectrum of the low-energy mode (the pole of the Green's function) does not change for $1<n<2$. This is because the flatbands cannot earn any kinetic or potential energy when $\Delta=0$ while ferrimagnetism is stabilized here to earn the total energy of electrons.

Next, we consider the case $\Delta>0$ with massive Dirac dispersions. In the limit $\omega / \Delta \rightarrow 0$, the sum of the second and third Lindhard functions in Eq. (C1) vanishes other than the term including $n_{\gamma, k \downarrow}$ again. We find the overall renormalization of the low-energy mode for $n=1+x$ as $\omega_{q}(x)=$ $\omega_{q}+\delta \omega_{q}(x)$, with

$$
\begin{aligned}
\delta \omega_{q}(x) & =-\frac{1}{2 S N} \sum_{k}\left|L_{k . q}\right|^{2} \frac{n_{c, k-q \uparrow}}{2 \Delta} \\
& =x\left\{4 j_{1} S\left[1+\Gamma_{1 q}\right]+8 j_{2} S\left[1-\Gamma_{2 q}\right]\right\} .
\end{aligned}
$$

$$
\begin{aligned}
& j_{1}=-\frac{1}{2 S^{2} N} \sum_{k} \frac{\cos ^{2} k_{x} \cos ^{2} k_{y}}{\Delta \varepsilon_{k}^{2}} \simeq-\frac{0.045}{S^{2} \Delta}, \\
& j_{2}=\frac{1}{4 S^{2} N} \sum_{k} \frac{\cos 2 k_{x} \cos ^{2} k_{y}}{\Delta \varepsilon_{k}^{2}} \simeq-\frac{0.017}{S^{2} \Delta} .
\end{aligned}
$$

The corrections appear to be negligibly small, amounting to only a few percent change of exchange couplings for electron doping in the physically accessible range $1<n<2$.

\section{APPENDIX D: HIDDEN SU (2) SYMMETRY}

To uncover the origin of the gapless mode discussed above, we note that the obtained spin-wave spectrum, Eq. (5) in the main text, is exactly the same as that of the following spinonly model on the square lattice of iron sites for local spin moments:

$$
\mathcal{H}_{\text {spin }}=J_{1} \sum_{\langle i j\rangle}\left[S_{i}^{x} S_{j}^{x}+S_{i}^{y} S_{j}^{y}-S_{i}^{z} S_{j}^{z}\right]-J_{2} \sum_{\langle i j\rangle\rangle} \vec{S}_{i} \cdot \vec{S}_{j},
$$

with anisotropic NN $\left(J_{1}\right)$ and isotropic NNN $\left(J_{2}\right)$ couplings. It is straightforward to verify that the model Eq. (D1) hosts a hidden spin-rotation symmetry and can be mapped to the isotropic ferromagnetic Heisenberg model by rotating spins sitting on the odd rows along the $x$ direction (corresponding to one set of staggered sublattice of the square lattice of iron sites) around the $z$ axis by an angle $\pi\left(S_{i}^{x} \rightarrow-S_{i}^{x}, S_{i}^{y} \rightarrow\right.$ $\left.-S_{i}^{y}, S_{i}^{z}=S_{i}^{z}\right)$. This site-dependent spin rotation also shifts $q=(\pi, 0) \rightarrow(0,0)$. This explains the origin and the location of the gapless Nambu-Goldstone mode accompanied by the spontaneous symmetry breaking of this hidden $S U(2)$ symmetry. Indeed, the same spin rotation for fermionic operators $\left(d_{i x z(y z) \sigma} \rightarrow \sigma d_{i x z(y z) \sigma}, c_{i \sigma} \rightarrow \sigma c_{i \sigma}\right)$ at every second row, together with local moments, maps the hopping Hamiltonian [see Eq. (2) in the main text] to the $S U(2)$-invariant form and leaves the local one [Eq. (3)] unchanged. However, we point out that this emergent spin-rotation symmetry is only approximate in reality and exists only in the extreme $\lambda \rightarrow \infty$ limit considered here, and thus the ferrimagnetic order should be pinned along the $z$ direction.
[1] H. Boschker and J. Mannhart, Quantum-matter heterostructures, Ann. Rev. Condens. Matter Phys. 8, 145 (2017).

[2] H. Y. Hwang, Y. Iwasa, M. Kawasaki, B. Keimer, N. Nagaosa, and Y. Tokura, Emergent phenomena at oxide interfaces, Nat. Mater. 11, 103 (2012).

[3] H. Takagi and H. Y. Hwang, An emergent change of phase for electronics, Science 327, 1601 (2010).

[4] D. G. Schlom, L.-Q. Chen, X. Pan, A. Schmehl, and M. A. Zurbuchen, A thin film approach to engineering functionality into oxides, J. Am. Ceram. Soc. 91, 2429 (2008).

[5] A. Chen, J.-M. Hu, P. Lu, T. Yang, W. Zhang, L. Li, T. Ahmed, E. Enriquez, M. Weigand, Q. Su, H. Wang, J.-X. Zhu, J. L. MacManus-Driscoll, L.-Q. Chen, D. Yarotski, and Q. Jia, Role of scaffold network in controlling strain and functionalities of nanocomposite films, Sci. Adv. 2, e1600245 (2016).
[6] A. Ohtomo and H. Y. Hwang, A high-mobility electron gas at the $\mathrm{LaAlO}_{3} / \mathrm{SrTiO}_{3}$ heterointerface, Nature 427, 423 (2004).

[7] N. Reyren, S. Thiel, A. D. Caviglia, L. F. Kourkoutis, G. Hammerl, C. Richter, C. W. Schneider, T. Kopp, A.-S. Rüetschi, D. Jaccard, M. Gabay, D. A. Muller, J.-M. Triscone, and J. Mannhart, Superconducting interfaces between insulating oxides, Science 317, 1196 (2007).

[8] M. Ben Shalom, M. Sachs, D. Rakhmilevitch, A. Palevski, and Y. Dagan, Tuning Spin-Orbit Coupling and Superconductivity at the $\mathrm{SrTiO}_{3} / \mathrm{LaAlO}_{3}$ Interface: A Magnetotransport Study, Phys. Rev. Lett. 104, 126802 (2010).

[9] Q. H. Wang, K. Kalantar-Zadeh, A. Kis, J. N. Coleman, and M. S. Strano, Electronics and optoelectronics of two-dimensional transition metal dichalcogenides, Nat. Nanotechnol. 7, 699 (2012). 
[10] C. L. Kane and E. J. Mele, Quantum Spin Hall Effect in Graphene, Phys. Rev. Lett. 95, 226801 (2005).

[11] K.-I. Kobayashi, T. Kimura, H. Sawada, K. Terakura, and Y. Tokura, Room-temperature magnetoresistance in an oxide material with an ordered double-perovskite structure, Nature 395, 677 (1998).

[12] A. Maignan, B. Raveau, C. Martin, and M. Hervieu, Large intragrain magnetoresistance above room temperature in the double perovskite $\mathrm{Ba}_{2} \mathrm{FeMoO}_{6}$, J. Solid State Chem. 144, 224 (1999).

[13] R. P. Borges, R. M. Thomas, C. Cullinan, J. M. D. Coey, R. Suryanarayanan, L. Ben-Dor, L. Pinsard-Gaudart, and A. Revcolevschi, Magnetic properties of the double perovskites $\mathrm{A}_{2} \mathrm{FeMoO}_{6} ; \mathrm{A}=\mathrm{Ca}, \mathrm{Sr}, \mathrm{Ba}$, J. Phys.: Condens. Matter 11, L445 (1999).

[14] X. Yuan, M. Xu, and Y. Chen, Magnetic properties and magnetoresistance effect of $\mathrm{Pb}_{2} \mathrm{FeMoO}_{6}$, Appl. Phys. Lett. 103, 052411 (2013).

[15] D. D. Sarma, P. Mahadevan, T. Saha-Dasgupta, S. Ray, and A. Kumar, Electronic Structure of $\mathrm{Sr}_{2} \mathrm{FeMoO}_{6}$, Phys. Rev. Lett. 85, 2549 (2000).

[16] Z. Fang, K. Terakura, and J. Kanamori, Strong ferromagnetism and weak antiferromagnetism in double perovskites: $\mathrm{Sr}_{2} \mathrm{Fe} \mathrm{O}_{6}(M=\mathrm{Mo}, \mathrm{W}$, and Re), Phys. Rev. B 63, 180407(R) (2001).

[17] I. V. Solovyev, Electronic structure and stability of the ferrimagnetic ordering in double perovskites, Phys. Rev. B 65, 144446 (2002).

[18] T. Saitoh, M. Nakatake, A. Kakizaki, H. Nakajima, O. Morimoto, S. Xu, Y. Moritomo, N. Hamada, and Y. Aiura, Half-metallic density of states in $\mathrm{Sr}_{2} \mathrm{FeMoO}_{6}$ due to Hund's rule coupling, Phys. Rev. B 66, 035112 (2002).

[19] A. Chattopadhyay and A. J. Millis, Theory of transition temperature of magnetic double perovskites, Phys. Rev. B 64, 024424 (2001).

[20] G. Jackeli, Microscopic model, spin-wave theory, and competing orders in double perovskites, Phys. Rev. B 68, 092401 (2003).

[21] O. Erten, O. N. Meetei, A. Mukherjee, M. Randeria, N. Trivedi, and P. Woodward, Theory of Half-Metallic Ferrimagnetism in Double Perovskites, Phys. Rev. Lett. 107, 257201 (2011).

[22] C. Du, R. Adur, H. Wang, A. J. Hauser, F. Yang, and P. C. Hammel, Control of Magnetocrystalline Anisotropy by Epitaxial Strain in Double Perovskite $\mathrm{Sr}_{2} \mathrm{FeMoO}_{6}$ films, Phys. Rev. Lett. 110, 147204 (2013).

[23] A. J. Hauser, R. E. A. Williams, R. A. Ricciardo, A. Genc, M. Dixit, J. M. Lucy, P. M. Woodward, H. L. Fraser, and F. Yang, Unlocking the potential of half-metallic $\mathrm{Sr}_{2} \mathrm{FeMoO}_{6}$ films through controlled stoichiometry and double-perovskite ordering, Phys. Rev. B 83, 014407 (2011).

[24] G. Chen, R. Pereira, and L. Balents, Exotic phases induced by strong spin-orbit coupling in ordered double perovskites, Phys. Rev. B 82, 174440 (2010).

[25] W. M. H. Natori, E. C. Andrade, E. Miranda, and R. G. Pereira, Chiral Spin-Orbital Liquids with Nodal Lines, Phys. Rev. Lett. 117, 017204 (2016).

[26] J. Romhányi, L. Balents, and G. Jackeli, Spin-Orbit Dimers and Noncollinear Phases in $d^{1}$ Cubic Double Perovskites, Phys. Rev. Lett. 118, 217202 (2017).
[27] G. Chen and L. Balents, Spin-orbit effects in $\mathrm{Na}_{4} \mathrm{Ir}_{3} \mathrm{O}_{8}$ : A hyper-kagome lattice antiferromagnet, Phys. Rev. B 78, 094403 (2008).

[28] G. Jackeli and G. Khaliullin, Mott Insulators in the Strong SpinOrbit Coupling Limit: From Heisenberg to a Quantum Compass and Kitaev Models, Phys. Rev. Lett. 102, 017205 (2009).

[29] A. M. Cook and A. Paramekanti, Double Perovskite Heterostructures: Magnetism, Chern Bands, and Chern Insulators, Phys. Rev. Lett. 113, 077203 (2014).

[30] A. M. Cook, Individual band with higher Chern numbers in double perovskite (001) monolayers, Phys. Rev. B 94, 205135 (2016).

[31] S. Baidya, U. V. Waghmare, A. Paramekanti, and T. Saha-Dasgupta, Controlled confinement of half-metallic twodimensional electron gas in $\mathrm{BaTiO}_{3} / \mathrm{Ba}_{2} \mathrm{FeReO}_{6} / \mathrm{BaTiO}_{3}$ heterostructures: A first-principles study, Phys. Rev. B 92, 161106(R) (2015).

[32] G. Jackeli and G. Khaliullin, Magnetically Hidden Order of Kramers Doublets in $d^{1}$ Systems: $\mathrm{Sr}_{2} \mathrm{VO}_{4}$, Phys. Rev. Lett. 103, 067205 (2009).

[33] A. Abragam and B. Bleaney, Electron Paramagnetic Resonance of Transition Ions (Clarendon Press, Oxford, 1970).

[34] M. G. Yamada, M. Oshikawa, and G. Jackeli, Emergent SU(4) Symmetry in $\alpha-\mathrm{ZrCl}_{3}$ and Crystalline Spin-Orbital Liquids, Phys. Rev. Lett. 121, 097201 (2018).

[35] All matrix elements of the total magnetic moment $\vec{M}=2 \vec{s}-\vec{l}$ in the pure $j=l+s=3 / 2$ manifold are strictly zero and the corresponding $g$ factor vanishes $(g=0)$. However, the covalence mixing between the $d$ orbitals and the oxygen $p$ orbitals gives rise to a finite but small $g$ factor $(g \ll 1)$ [33].

[36] Z. Wang, Z. Zhong, X. Hao, S. Gerhold, B. Stöger, M. Schmid, J. Sánchez-Barriga, A. Varykhalov, C. Franchini, K. Held, and U. Diebold, Anisotropic two-dimensional electron gas at $\mathrm{SrTiO}_{3}(110)$, Proc. Natl. Acad. Sci. 111, 3933 (2014).

[37] M. Garnica, D. Stradi, S. Barja, F. Calleja, C. Díaz, M. Alcamí, N. Martín, A. L. Vázquez de Parga, F. Martín, and R. Miranda, Long-range magnetic order in a purely organic 2D layer adsorbed on epitaxial graphene, Nat. Phys. 9, 368 (2013).

[38] M. G. Yamada, T. Soejima, N. Tsuji, D. Hirai, M. Dincă, and H. Aoki, First-principles design of a half-filled flat band of the kagome lattice in two-dimensional metal-organic frameworks, Phys. Rev. B 94, 081102(R) (2016).

[39] E. H. Lieb, Two Theorems on the Hubbard Model, Phys. Rev. Lett. 62, 1201 (1989).

[40] A. Mielke, Ferromagnetic ground states for the Hubbard model on line graphs, J. Phys. A: Math. Gen. 24, L73 (1991).

[41] H. Tasaki, Ferromagnetism in the Hubbard Models with Degenerate Single-Electron Ground States, Phys. Rev. Lett. 69, 1608 (1992).

[42] E. Wigner, On the interaction of electrons in metals, Phys. Rev. 46, 1002 (1934).

[43] D. Sánchez, J. A. Alonso, M. García-Hernández, M. J. Martínez-Lope, M. T. Casais, J. L. Martínez, and M. T. Fernández-Díaz, Electron and hole doping effects in $\mathrm{Sr}_{2} \mathrm{FeMoO}_{6}$ double perovskites, J. Magn. Magn. Mater. 272-276, 1732 (2004).

[44] R. Chitra, T. Giamarchi, and P. Le Doussal, Pinned Wigner crystals, Phys. Rev. B 65, 035312 (2001). 